\title{
APPROXIMATE ANALYSIS OF STRUCTURES \\ IN THE PRESENCE OF MODERATELY LARGE CREEP DEFORMATIONS*
}

\author{
$\mathrm{By}$ \\ N. J. HOFF \\ Polytechnic Institute of Brooklyn
}

1. Introduction. When creep strains of the order of magnitude of one or two percent develop during the lifetime of a structure, it is often permissible to disregard the primary phase of the creep deformations and to base the analysis solely on the secondary or steady phase of creep. For the metals used in structures the experimentally established secondary creep law is generally given in the form

$$
e_{11}=K a_{11}^{n},
$$

where $e_{11}$ is the tensile strain rate caused by uniaxial tension in direction $1, \sigma_{11}$ is the corresponding tensile stress, and $K$ and $n$ are constants. When the creep strains are large (of the order of magnitude of 0.01 ), the elastic deformations can often be neglected in the calculations, as will be demonstrated by means of an example. Thus the limiting state of stress and strain approached as the creep strain becomes large as compared to the elastic strain can be determined on the basis of a simple non-linear stress-strain rate law.

It is believed that structural analyses based on the assumptions stated are satisfactory for supersonic guided missiles whose surface is heated to high temperatures by the air flow. As guided missiles are generally used only for a single flight and not over long periods of time like piloted airplanes, their structure can be permitted to undergo large permanent deformations.

2. The elastic analogue. It will now be shown that the stress distribution in a body whose deformations are governed by a generalized version of the non-linear creep law of Eq. 1 is the same as that in a non-linear perfectly elastic body provided the elastic stress-strain law and the boundary conditions are suitably chosen. Following Prager's suggestions for the representation of the stress-strain laws of strain-hardening materials [1] $\dagger$, the uniaxial stress-strain rate law of Eq. 1 is generalized to read

where

$$
E=f\left(J_{2}, J_{3}\right)\left[p\left(J_{2}, J_{3}\right) T+q\left(J_{2}, J_{3}\right) S^{\prime}\right],
$$

$$
T=S^{\prime 2}-(2 / 3) J_{2} I,
$$

$E$ is the strain rate tensor, $S^{\prime}$ the stress deviation tensor, and $I$ the identity tensor. The typical component of the stress deviation tensor is defined as

$$
s_{i j}^{\prime}=s_{i i}-(1 / 3) s_{k k} \delta_{i i},
$$

*Received June 15, 1953. Part of the analysis described in this article was performed under Contract No. AF33(616)-116 sponsored at the Polytechnic Institute of Brooklyn by the Wright Air Development Center, Air Research and Development Command, of the U.S. Air Force. The author is grateful to the U.S. Air Force for permission to publish this article. He also acknowledges his indebtedness to Professor W. Prager of Brown University for his detailed suggestions regarding the formulation of stress-strain laws in triaxial states of stress and the statement of the analogues; and to Professor Frederick V. Pohle of the Polytechnic Institute of Brooklyn who was kind enough to check the calculations.

$\nmid$ Numbers in brackets refer to the References. 
and $s_{i j}$ is the typical component of the stress tensor. The first invariant of the stress deviation naturally vanishes:

$$
J_{1}=s_{i i}^{\prime}=0 .
$$

The second and third invariants are defined as

$$
\begin{aligned}
& J_{2}=(1 / 2) s_{i j}^{\prime} s_{i i}^{\prime}, \\
& J_{3}=(1 / 3) s_{i j}^{\prime} s_{j k}^{\prime} s_{k i}^{\prime} .
\end{aligned}
$$

In Eq. 2 the symbols $p\left(J_{2}, J_{3}\right)$ and $q\left(J_{2}, J_{3}\right)$ denote polynomial functions, and $f\left(J_{2}, J_{3}\right)$ an arbitrary function of $J_{2}$ and $J_{3}$. These functions must be determined from empirical data to be obtained from creep tests. Eq. 2 is meant for use only when the strain is small as compared to unity (say 0.01 ); under such conditions it implies that the creep deformations are inextensional $\left(e_{i i}=0\right)$.

If a body which follows this stress-strain rate law is subjected to given body forces $\varphi_{i}(x, t)$, (where $x$ is understood to represent the three Cartesian coordinates of space) and to given surface tractions $T_{i}(x, t)$ on a portion $S_{1}$ of its surface while the points on the remainder $S_{2}$ of the surface are slowly displaced with given velocities $V_{i}(x, t)$ (so slowly that the resulting inertia forces are small as compared to the forces corresponding to the stresses $S$ and surface tractions $T$ ), at a generic instant $t$ the stress field $s_{i i}(x, t)$ and the velocity field $v_{i}(x, t)$ throughout the body must satisfy the following equations:

$$
\begin{gathered}
\left(\partial s_{i j} / \partial x_{i}\right)+\varphi_{i}=0 \\
\left(\partial v_{i} / \partial x_{i}\right)+\left(\partial v_{i} / \partial x_{i}\right)=2 f\left(J_{2}, J_{3}\right)\left[p\left(J_{2}, J_{3}\right) t_{i i}+q\left(J_{2}, J_{3}\right) s_{i j}^{\prime}\right] .
\end{gathered}
$$

The three equilibrium equations (4) and the six stress-strain rate equations (5), together with the boundary conditions

$$
\begin{aligned}
& s_{i j} n_{i}=T_{i} \quad \text { on } \quad S_{1}, \\
& v_{i}=V_{i} \quad \text { on } S_{2} \text {, }
\end{aligned}
$$

define the stress and velocity fields in the body.

The analoguous perfectly elastic body is required to follow the stress-strain law

$$
E^{*}=f\left(J_{2}, J_{3}\right)\left[p\left(J_{2}, J_{3}\right) T+q\left(J_{2}, J_{3}\right) S^{\prime}\right]
$$

in which the only new symbol, $E^{*}$, represents the strain tensor whose typical component is $\epsilon_{i j}$. The body forces and the surface tractions are kept unchanged but the velocities $V_{i}(x, t)$ prescribed on surface $S_{2}$ are replaced by displacements $U_{i}(x, t)$ equal to $V_{i}(x, t)$ in magnitude and direction. Under these conditions the displacement field $u_{i}(x, t)$ and the stress field $s_{i j}(x, t)$ in the elastic body are defined by Eqs. 4 to 6 if $v_{i}(x, t)$ is replaced by $u_{i}(x, t)$ and $V_{i}(x, t)$ by $U_{i}(x, t)$. Consequently to any solution of the elastic problem there corresponds a solution of the creep problem and the stress distribution is the same in the two solutions.

Similar results were published earlier for linear visco-elastic materials by Alfrey [2] and Tsien [3].

3. Pin-jointed truss. As an example for the use of the analogue the stresses will now be calculated in the bars of the pin-jointed framework shown in Fig. 1. One end of each 


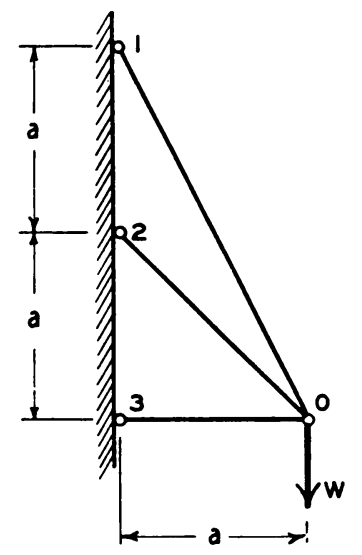

Fig. 1. Pin-Jointed Framework

bar is attached to a rigid wall. Bar $0-3$, which is in compression when the load $W$ is applied to joint $O$, is assumed to be perfectly rigid and braced in the direction perpendicular to the plane of the truss so as to prevent its buckling. The material of the other two bars is subject to creep in accordance with the law

$$
e=(\sigma / \lambda)^{n},
$$

where $e$ is the rate of creep in uniaxial tension, $\sigma$ the tensile stress, and $\lambda$ and $n$ are constants. Because of the analogue the creep problem can be replaced by a problem in non-linear elasticity with the stress-strain law.

$$
\epsilon=(\sigma / \lambda)^{n},
$$

where $\epsilon$ is the tensile strain.

It might be mentioned here that the behavior of pin-jointed structures was investigated by Meacham [4] on the assumption of a linear creep law.

3a. Load $W$ prescribed. When the load $W$ is prescribed, the stress distribution can be calculated from the requirement that the complementary energy stored in the bars must be a minimum. If the force in bar 0-2 is designated as $X$, it follows from the conditions of equilibrium that the forces in the three bars are

$$
F_{0-1}=(\sqrt{5} / 2) W-(\sqrt{10} / 4) X, \quad F_{0-2}=X, \quad F_{0-3}=-(1 / 2) W-(\sqrt{2} / 4) X
$$

As the complementary energy per unit volume $\left(U^{\prime} / V\right)$ is defined as

$$
\left(U^{\prime} / V\right)=\int_{0}^{\sigma} \epsilon d \sigma
$$

substitution from Eq. 9 and integration yield

$$
\left(U^{\prime} / V\right)=[\lambda /(n+1)](\sigma / \lambda)^{n+1} .
$$

With $A$ designating the common cross-sectional area of the two elastic bars, the strain energy stored in the system becomes

$$
U^{\prime}=\left[(n+1) \sigma \lambda^{n} A^{n}\right]^{-1}\left\{\sqrt{5}[(\sqrt{5} / 2) W-(\sqrt{10} / 4) X]^{n+1}+\sqrt{2} X^{n+1}\right\} .
$$


In accordance with the complementary energy principle the differential coefficient of $U^{\prime}$ with respect to $X$ must vanish. After some manipulations this requirement can be written in the form

$$
(X / W)=(\sqrt{5} / 2)\left[(4 / 5)^{1 / n}+(\sqrt{10} / 4)\right]^{-1} .
$$

After $(X / W)$ is computed from Eq. 14 for any given $n$, the values of $F_{0-1}$ and $F_{0-2}$ can be calculated from Eqs. 10. The results of such computations are plotted in Fig. 2.

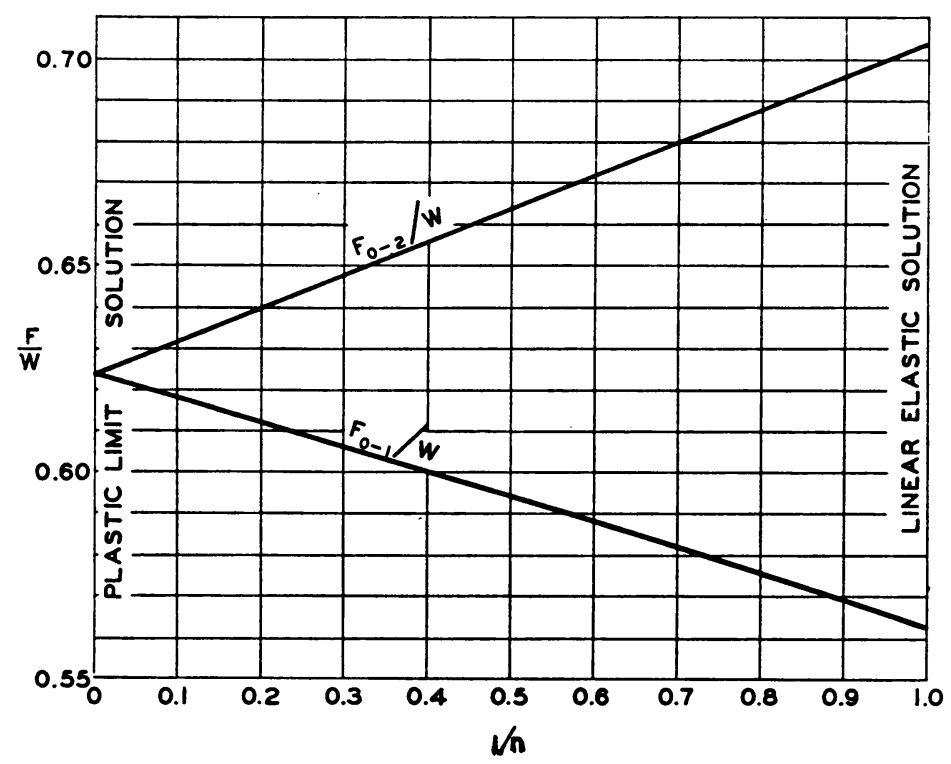

Fig. 2. Forces in Bars of Pin-Jointed Framework

When $n=1$, the linear elastic solution is obtained; and when $n \rightarrow \infty$, the solution corresponds to the principles of limit analysis.

$3 \mathrm{~b}$. Velocity $V_{0}$ prescribed. If the velocity $V_{0}$ of joint 0 is prescribed in the creep problem rather than the load $W$, in the analogous elastic problem the elastic displacement $U_{0}$ of joint 0 must be given. The potential $V^{\prime}$ of the unknown reaction force $R$ at 0 is

$$
-V^{\prime}=R U_{0}
$$

with both $R$ and $U_{0}$ considered positive when directed downward. The forces in the bars caused by the unknown reaction $R$ can be calculated from Eqs. 10 if $W$ is replaced by $R$. The complementary energy can be obtained in a similar manner from Eq. 13 . The compatibility conditions are in this case

$$
\begin{aligned}
& \partial\left(U^{\prime}+V^{\prime}\right) / \partial X=0, \\
& \partial\left(U^{\prime}+V^{\prime}\right) / \partial R=0 .
\end{aligned}
$$

As a consequence of the first of these equations Eq. 14 again holds provided $W$ is replaced by $R$. It follows from the second equation that

$$
(R / A)=(2 / \sqrt{5})\left[(4 / 5)^{1 / n}+(\sqrt{10} / 4)\right] \lambda\left(U_{0} / 2 a\right)^{1 / n} .
$$


3c. More accurate solution of the creep problem. The problem just discussed is so simple that it could have been solved on the basis of geometric considerations of the deformations without recourse to energy methods. For the same reason it can also be analyzed in the case when the deformations are governed by a more complex creep law than the one represented by Eq. 8. Such a more complete analysis will now be presented in order to show that the initial (linear) elastic stress distribution in an actual structure is rapidly replaced by a distribution that, for all practical purposes, is identical with the stress distribution derived from the (non-linear) elastic analogue.

The creep law will be assumed as

$$
e=(1 / E)(d \sigma / d t)+(\sigma / \lambda)^{n},
$$

where $t$ is time. If $e_{1}$ is the strain rate in bar $0-1$, the rate of elongation $d \Delta L_{1} / d t$ in the bar is

Similarly in bar 0-2

$$
d \Delta L_{1} / d t=\sqrt{5} a e_{1} .
$$

$$
d \Delta L_{2} / d t=\sqrt{2} a e_{2} .
$$

The geometric condition imposed upon these deformations can be derived from Fig. 3 .

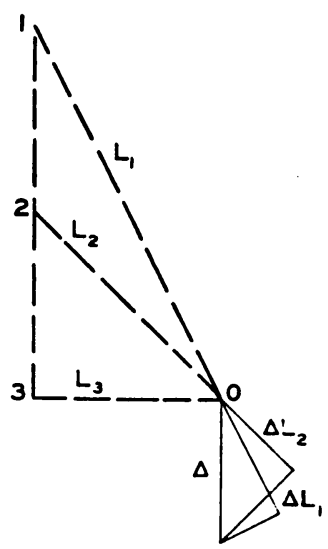

FIg. 3. Displacement of Joint $O$

When the length of bar 0-1 is increased from $L_{1}$ to $L_{1}+\Delta L_{1}$ the length of bar 0-3 remains $L_{3}$ because this bar was assumed to be perfectly rigid. The new position of joint 0 can then be found by drawing an arc of a circle from point 1 with a radius $L_{1}+\Delta L_{1}$, a second arc of a circle from point 3 with a radius $L_{3}$, and by determining the point of intersection of the two arcs. When the deformations are small, the arcs of circles can be replaced by straight line segments perpendicular to the original directions of bars $0-1$ and $0-3$, respectively. This was done in the figure and the vertical displacement $\Delta$ of joint 0 was obtained as

$$
\Delta=(\sqrt{5} / 2) \Delta L_{1} .
$$

The same vertical displacement must result if the geometric construction is carried out for bar 0-2. Hence

$$
\Delta=\sqrt{2} \Delta L_{2} .
$$


When these two equations are differentiated with respect to time and their right-hand members are equated to each other, the following condition is obtained:

$$
(\sqrt{5} / 2) d \Delta L_{1} / d t=\sqrt{2} d \Delta L_{2} / d t .
$$

Substitutions from Eqs. 18 and 19 yield

$$
(5 / 4)\left[(1 / E)\left(d \sigma_{1} / d t\right)+\left(\sigma_{1} / \lambda\right)^{n}\right]=(1 / E)\left(d \sigma_{2} / d t\right)+\left(\sigma_{2} / \lambda\right)^{n} .
$$

When the load $W$ is prescribed at joint 0 , equilibrium requires that

$$
(W / A)=(2 / \sqrt{5}) \sigma_{1}+(1 / \sqrt{2}) \sigma_{2} .
$$

Solution of this equation for $\sigma_{2}$, substitution in Eq. 22, and manipulations result in the differential equation

$$
(d x / d t)=K\left[(1-b x)^{n}-(c x)^{n}\right],
$$

where

$$
\begin{gathered}
x=\left(A \sigma_{1} / W\right), \quad b=(4 / 5)^{1 / 2}, \quad c=(5 / 4)^{1 / n}(1 / 2)^{1 / 2}, \\
K=\left[2^{n / 2}(W / A \lambda)^{n-1}(E / \lambda)\right] /\left[(5 / 4)+(8 / 5)^{1 / 2}\right] .
\end{gathered}
$$

After separation of the variables and integration the following solution is obtained:

$$
t=(1 / K) \int_{x_{0}}^{x} d x /\left[(1-b x)^{n}-(c x)^{n}\right] .
$$

A numerical example was worked out in which the material of the bars was 52S-H38 alluminum alloy. At $400^{\circ} \mathrm{F}$ the material constants can be taken as $n=5$,

$$
\lambda=25,000 \mathrm{hr}^{1 / 5} \mathrm{lb} \text {. per sq. in. }
$$

on the basis of creep tests carried out by Dorn and Tietz [5]. From Table 3.1211(b) in ANC-5 [6] Young's modulus can be estimated as $9 \times 10^{6} \mathrm{lb}$. per sq. in. With these values one obtains $K=487$ per hour if the load is assumed to be $22,000 \mathrm{lbs}$. The load is applied at $t=0$ and dynamic effects are disregarded. Initially the fully (linearly) elastic solution must prevail; correspondingly $x=0.56233$ when $t=0$. The state of fully developed creep is reached only as $t$ approaches infinity; then, in the limit, $x=0.61206$. Evaluation of the integral in Eq. 26 yielded corresponding values of $x$ and $t$ which were plotted in Fig. 4 . It can be seen from the figure that after the lapse of about 70 sec. $x$ is 0.61 which is only one-third of one percent less than the fully developed creep value 0.61206 ; (the difference between 0.61 and 0.61206 is about four percent of the difference between the elastic and the fully developed creep values). As the highest stress in any bar is about 15,000 lb. per sq. in. (in bar 0-2 at $t=0$ ), the maximum creep rate is about 0.0777 per hour. Hence the total creep strain developed in bar 0-2 in the first 70 sec. after the load is applied is less than 0.00151 . This should be compared with the maximum elastic strain in the bar which is 0.00167 .

Thus the conclusion is reached that in this problem in good approximation the state of fully developed creep is reached at a time when the creep strain is about equal to the elastic strain. The effect of the elastic stresses can therefore be neglected when the state of stress and strain is investigated at moderately large creep deformations (at creep strains of the order of magnitude of 0.01 ). 


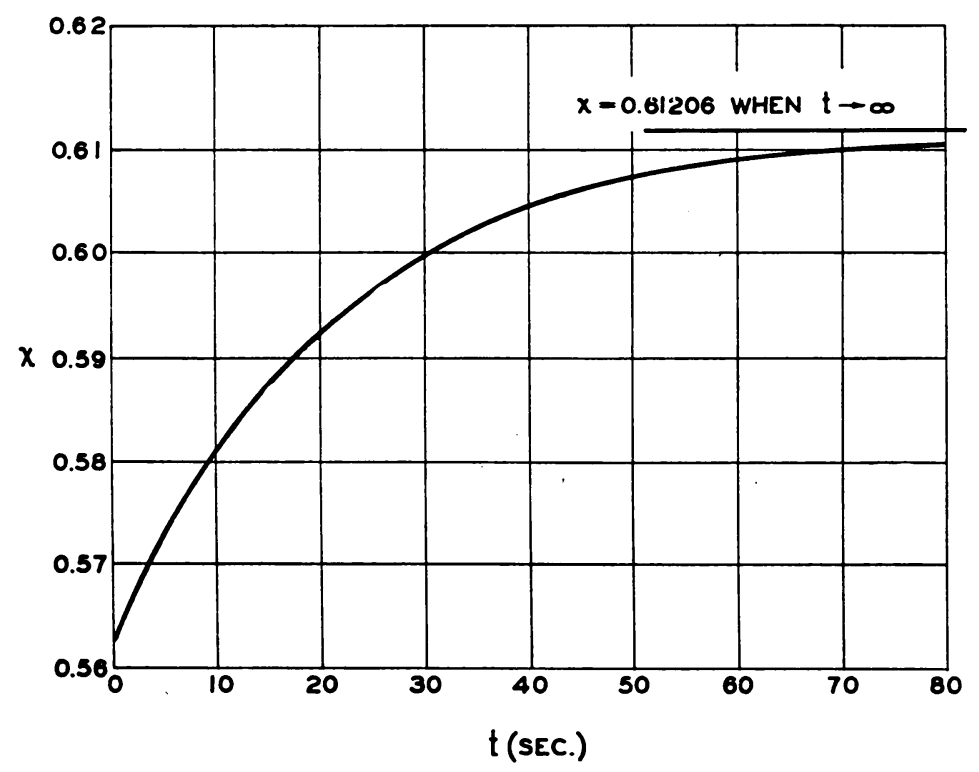

FIg. 4. Variation in Non-dimensional Force in Bar $O-1$ with Time

Finally it is of interest to check whether the time interval of 70 sec. is short as compared to the time needed for the necking and rupture of the most highly loaded bar. In an earlier paper [7] the author derived the formula

$$
t_{c r}=(1 / n) /\left(\sigma_{0} / \lambda\right)^{n},
$$

where $t_{c r}$ is the time needed for rupture and $\sigma_{0}$ is the engineering stress at the beginning of the creep test. It is reasonable to take $\sigma_{0}=14,100 \mathrm{lb}$. per sq. in. corresponding to the fully developed state of creep in bar $0-2$. With this value one obtains $t_{c r}=3.55$ hours. Thus there is a long period of time during which the fully developed creep solution is valid.

\section{REFERENCES}

(1) W. Prager, Strain hardening under combined stresses, J. Appl. Phys. 16, 837-840 (1945). See also M. Reiner, A mathematical theory of dilatancy, Amer. J. Math. 67, 350-362, (1945) and Clifford A. Truesdell, A program of physical research in classical mechanics, Zs. angew. Math. Phys. 32, 79-95, (1952).

(2) T. Alfrey, Non-homogeneous stresses in visco-elastic media, Q. Appl. Math. 2, 113-119 (1944).

(3) H. S. Tsien, A generalization of Alfrey's theorem for visco-elastic media, Q. Appl. Math. 8, 104-106, (1950).

(4) Robert C. Meacham, Theory of creep in pin-jointed structures, Proc. First U. S. National Congr. Appl. Mech., 1952, pp. 619-624.

(5) J. E. Dorn and T. E. Tietz, Creep and stress-rupture investigations on some aluminum alloy sheet metals, Proc. Amer. Soc. Test. Mat. 49, 815-831, (1949).

(6) Munitions Board-Aircraft Committee, ANC-5 Bulletin, Strength of metal aircraft elements, U. S. Government Printing Office, 1951.

(7) N. J. Hoff, The necking and the rupture of rods subjected to constant tensile loads, J. Appl. Mech. 20, 105-108 (1953). 